\title{
Multi-Photon Absorption in Metal-Organic Frameworks
}

\author{
Raghavender Medishetty, ${ }^{[[a]}$ Lydia Nemec, ${ }^{\dagger[b]}$ Venkatram Nalla, ${ }^{*[c]}$ Sebastian Henke, ${ }^{[d]}$ Marek Samoć, ${ }^{[e]}$ \\ Karsten Reuter, ${ }^{*[b]}$ Roland A. Fischer*[a]
}

\begin{abstract}
Multi-photon absorption (MPA) is among the most prominent nonlinear optical (NLO) effects and has applications, for example in telecommunications, defense, photonics and biomedicines. Established MPA materials include dyes, quantum dots, organometallics and conjugated polymers, most often dispersed in solution. We demonstrate how metal-organic frameworks (MOFs), a novel NLO solid-state materials class, can be designed for exceptionally strong MPA behavior. MOFs consisting of zirconiumand hafnium-oxo-clusters and featuring a chromophore linker based on the tetraphenylethene (TPE) molecule exhibit record high twophoton absorption (2PA) cross section values, up to $3600 \mathrm{GM}$. The unique modular building-block principle of MOFs allows enhancing and optimizing their MPA properties in a theory guided approach by combining tailored charge polarization, conformational strain, threedimensional arrangement and alignment of the chromophore linkers in the crystal.
\end{abstract}

Multi-photon absorption (MPA) is a process in which two or more photons are simultaneously absorbed by a material (Figure 1). MPA facilitates important applications, such as two-photon fluorescence microscopy ${ }^{[1]}$, three-dimensional (3D) optical data storage $^{[2]}$, optical limiting ${ }^{[3]}$, and 3D microfabrication ${ }^{[4]}$, to name a few. So far, the search for materials with high MPA cross section values has focused on organic dye molecules, polymers, organometallic compounds and nanoparticles. ${ }^{[5]}$ However, commonly used MPA materials are mainly usable only as low concentrated solutions $\left(\sim 10^{-2} \mathrm{~mol} / \mathrm{dm}^{3}\right)^{[5 a]}$ to avoid unwanted aggregation and concentration quenching of the MPA excited emission, along with low optical stability. ${ }^{[6]}$

[a] Dr. Raghavender Medishetty, Prof. Dr. Roland A. Fischer Chair of Inorganic and Metal-Organic Chemistry, Technische Universität München, Lichtenbergstraße 4, 85747 Garching, Germany.

E-mail: roland.fischer@tum.de

[b] Dr. Lydia Nemec, Prof. Dr. Karsten Reuter

Chair of Theoretical Chemistry, Technische Universität München, Lichtenbergstraße 4, 85747 Garching, Germany.

E-mail: karsten.reuter@ch.tum.de

[c] Dr. Venkatram Nalla

Centre for Disruptive Photonic Technologies, School of Physical and Mathematical Sciences, Nanyang Technological University, Singapore 637371, Singapore.

E-mail:VNalla@ntu.edu.sg

[d] Dr. Sebastian Henke

Department of Chemistry and Chemical Biology, Technische Universität Dortmund, Otto-Hahn-Straße 6, 44227 Dortmund, Germany.

[e] Prof. Dr. Marek Samoć

Advanced Materials Engineering and Modelling Group, Wroclaw University of Science and Technology, Wyb. Wyspiańskiego 27, PL50370, Wrocław, Poland.

$\dagger \quad$ These authors contributed equally to this work.

Supporting information for this article is given via a link at the end of the document.
A new material class, metal-organic frameworks (MOFs), offers an elegant way to overcome these challenges. MOFs consist of metal ion/cluster nodes bound to organic molecules called linkers and self-assembled into crystalline structures ${ }^{[7]}$ with unique packing of the linkers (Figure 2). MOFs have mainly been investigated in the context of gas storage, gas separation and catalysis. ${ }^{[8]}$ Intrinsic nonlinear optical (NLO) properties can be tuned in MOFs by choosing chromophores as linkers. ${ }^{[9]}$ Alternatively, MOFs can be loaded (by impregnation) with dyes to achieve NLO (extrinsic) properties and multi-photon pumped lasing. ${ }^{[10]}$ Particularly, the spatial arrangement, rigidification and a specific conformation of the linker, caused by the pinning of the linker between the metal nodes leads to materials which outperform any solid-state MPA material known so far. We identified five important design criteria to obtain MOFs with record high MPA cross section values.

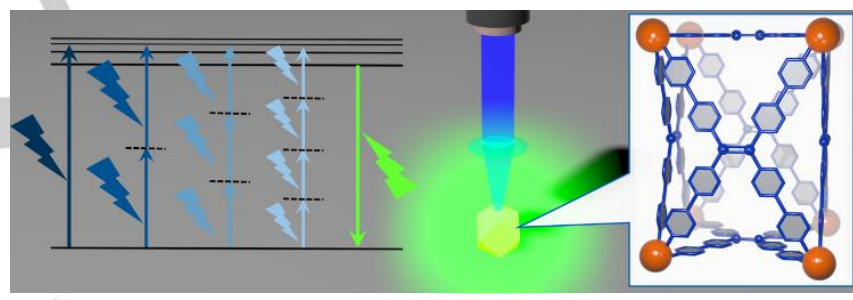

Figure 1. A scheme depicting the excitation of a metal-organic framework (MOF) crystal and the observation of multi-photon excited fluorescence. Middle: Emission of green fluorescence upon excitation of a yellow MOF crystal by a focused beam of a femtosecond laser. Left: Energy level diagram showing the simultaneous absorption of one to multiple photons and the green fluorescence emission from the excited state. Right: Schematic view of the MOF, with the metal-nodes (red spheres) interlinked by the chromophore linker (blue/ grey structures). Note, the conformation of the biphenyl units of the linker is simplified for clarity (see Supporting Information).

Our design concept for a perfect MOF for superior MPA applications follows several criteria that are similar to those employed for organic or organometallic NLO chromophore molecules: ${ }^{[5 b, 11]}$ a) molecular dipole or multipolar structural units for an enhanced polarization of the charge distribution, b) presence of planar structural motifs with long $\pi$-conjugation, $c$ ) high fluorescence quantum yield, d) narrow one-photon absorption (1PA) and two-photon (2PA) absorption bands, as well as significant wavelength differences between them, and e) alignment and high packing density (concentration $\mathrm{mol} / \mathrm{dm}^{3}$ ) of the NLO chromophore. A very desirable aspect and so far unresolved challenge to match the last criterion is the rational control of organization and arrangement of the chromophores, i.e. donor and acceptor units in 3D space. ${ }^{[12]}$ MOFs offer a unique access to a "targeted design" of this property.

In this work, we demonstrate how the 2PA cross section values of the parent tetrakis[4-(4-carboxyphenyl)phenyl]ethylene 


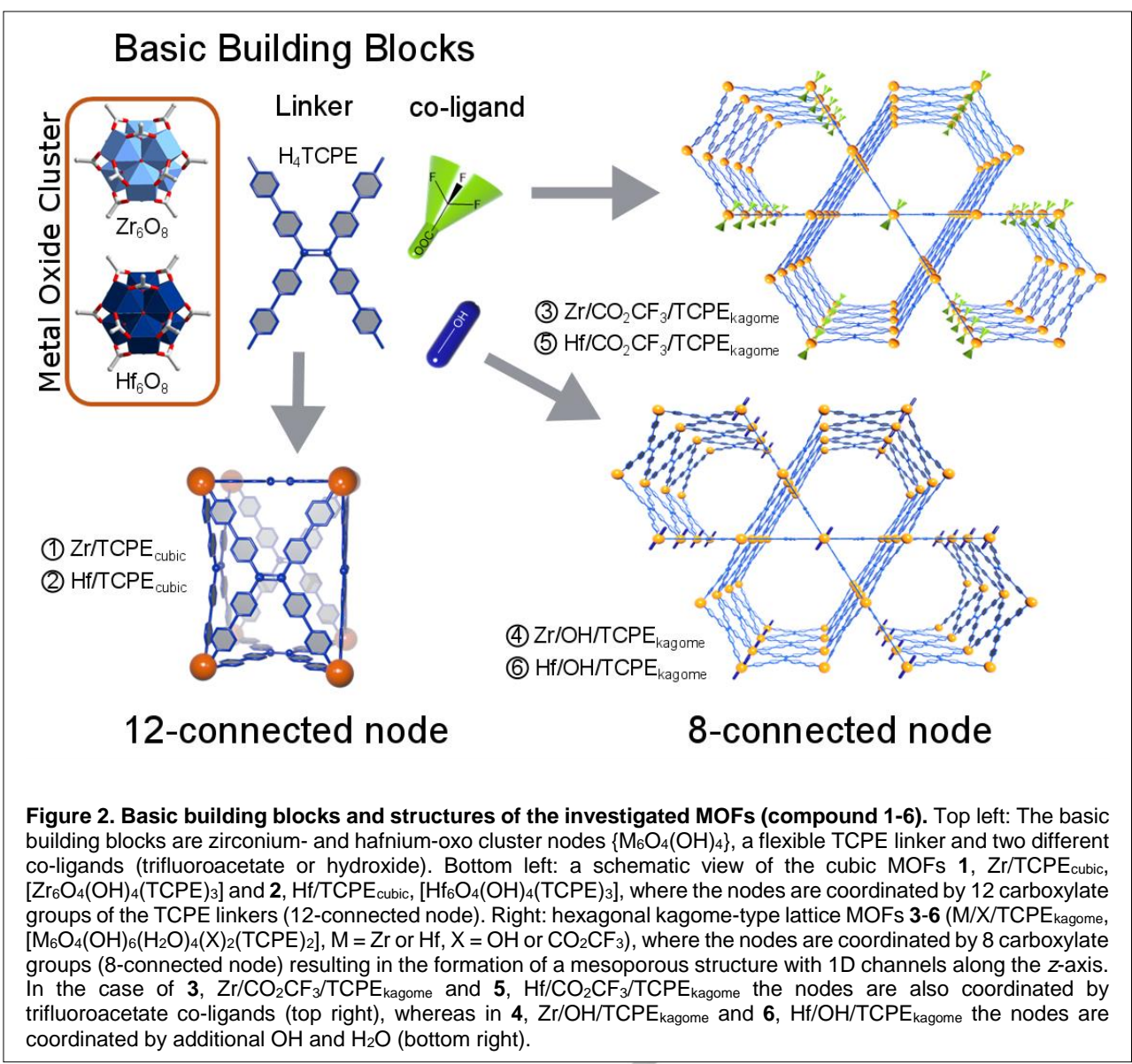

analogues (Figure 2; MOFs 2, 5, 6). In addition, we obtain electron-withdrawing TFAbonded structures $(\mathbf{3}, \mathbf{5})$ and TFA-hydrolyzed structures $(\mathbf{4}, \mathbf{6})$ to demonstrate the modulation of charge transfer. The hydrolyzed structures feature $\mathrm{OH}$ and $\mathrm{H}_{2} \mathrm{O}$ co-ligands instead of TFA.

There are various methods to investigate MPA optical properties. ${ }^{[5 a, 11,14]}$ We use multiphoton excited fluorescence (MPEF) measurements with an internal reference, in order to minimize the uncertainties due to scattering effects (the MOFs are suspended in rhodamine-B solutions; see Supporting Information, Section 2). ${ }^{[9 a]} \mathrm{We}$ start our investigations of MPA optical properties with the cubic structures 1 and 2, which are found to show 2PA action cross section values of $1035 \mathrm{GM}$ and $292 \mathrm{GM}$, respectively. The action cross section is a product of the absorption cross section $(\sigma)$ and the luminescence quantum yield (n). Figure 3 shows the measured 2PA (a), 3PA and 4PA

( $\mathrm{H}_{4}$ TCPE) NLO chromophore can be increased by two orders of magnitude by integrating them as TCPE linkers into a rigid MOF structure. Optimizing TCPE-based MOFs by synthesis to fulfill the above mentioned design criteria, we achieve record high 2PA action cross section values of $3582 \mathrm{GM}$ (GM abbreviates the unit Göppert-Mayer, $\left.1 \mathrm{GM}=10^{-50} \mathrm{~cm}^{4} \mathrm{~s}_{\text {photon }}{ }^{-1}\right)$.

To demonstrate the effect of the nature of the building blocks on the MPA efficiency, we choose zirconium- and hafnium-oxo clusters as inorganic nodes and trifluoroacetate (TFA) or hydroxide $(\mathrm{OH})$ as additional co-ligands (Figure 2). Employing these building blocks, we synthesize a series of chemically robust MOFs (1-6 in Figure 2) (see Supporting Information, Section 1). All six compounds feature nodes with an octahedral core unit $\left\{\mathrm{M}_{6} \mathrm{O}_{4}(\mathrm{OH})_{4}\right\}$, where $\mathrm{M}$ stands for the respective metal ion. The MOFs 1 and 2 crystallize in the cubic space group $P m-3 m$, where the 12-connected metal ion nodes are located at the cube corners and TCPE linkers cover the cubes faces. ${ }^{[9 b]}$ MOF 3-6 crystallize in the hexagonal space group $P 6 / \mathrm{mmm}$ and contain three 8connected nodes and six highly strained TCPE linkers per unit cell forming a 3D-kagome lattice. This structural arrangement leads to one of the largest known 1D open channels among MOFs. ${ }^{[13]}$ Nevertheless, a comparably dense packing of the TCPE can still be achieved, ${ }^{[9 c]}$ leading to chromophore linker concentrations of $0.52 \mathrm{~mol} / \mathrm{dm}^{3}(\mathbf{1}, \mathbf{2})$ and $0.28 \mathrm{~mol} / \mathrm{dm}^{3}(\mathbf{3}-\mathbf{6})$. The electrophilic charge polarization influence of the node on the chromophore linker is explored by including two series of MOFs, Zr-based nodes (Figure 2; MOFs 1, 3, 4) and their isostructural Hf- (b) action cross section values of 1-6. For comparison, a sample of the parent chromophore $\mathrm{H}_{4}$ TCPE exhibits a weak 2PA action cross section of $55 \mathrm{GM}$, while its 3PA is already too weak to be measured (Supporting Information, Table S5). The two MOFs 3 and $\mathbf{4}$ give the strongest action cross section values $\left(\eta \sigma_{2}=3582\right.$ GM and $2590 \mathrm{GM}$, respectively) ever found for any solid MPA material in the literature. ${ }^{[15]}$ The $\mathrm{Zr}$-based materials 1, $\mathbf{3}$ and $\mathbf{4}$ show higher 2PA, 3PA and 4PA action cross section values than their respective $\mathrm{Hf}$ analogues. Although the cubic MOFs $\mathbf{1}$ and 2 have a higher TCPE concentration, they show reduced, however, still very high 2PA action cross section values compared to dyes, quantum dots, porphyrins and other MOFs (see Supporting Information, Section 3); the same trend holds for the 3PA and 4PA (Figure 3b).

For a better understanding of this discovery, we perform electronic structure calculations on the level of time-dependent density functional theory (TDDFT) ${ }^{[16]}$ (see Supporting Information, Section 4) using the long-range corrected hybrid functional camB3LYP. ${ }^{[17]}$ We systematically investigate the origin of the massively increased 2PA cross section by exploring the influence of charge transfer, polarization and structural arrangement on the non-linear optical properties.

First, we study the effect of the metal-oxo cluster node and co-ligands on the charge transfer between the TCPE linker and the node by calculating Hirshfeld charges. ${ }^{[18]}$ For the two cubic MOFs 1 and 2, we obtain a charge transfer of $1 e^{-}$per linker, 

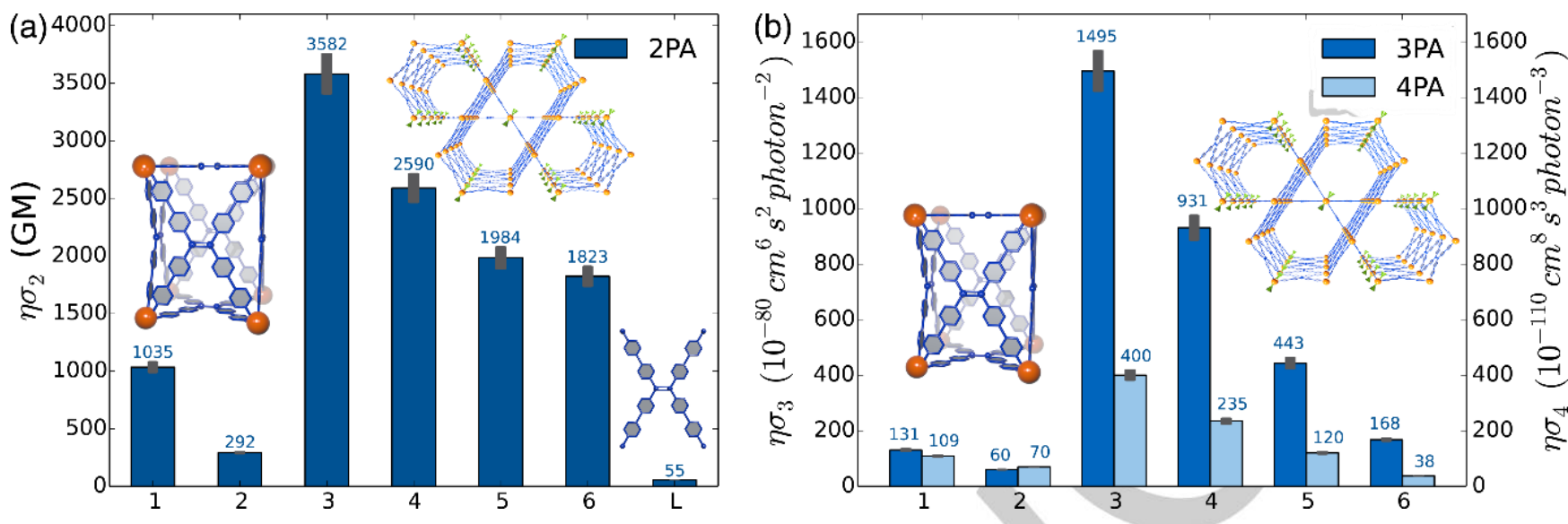

Figure 3. MPA action cross section values of MOFs 1-6 and $\mathrm{H}_{4}$ TCPE (L). (a) Comparison of $2 \mathrm{PA}$ cross section values $\eta \sigma_{2}$ (dark blue) of MOFs 1-6 (Figure 2 ) with $\mathrm{H}_{4} \mathrm{TCPE}\left(1 \mathrm{GM}=10^{-50} \mathrm{~cm}^{4} \mathrm{~s}\right.$ photon $\left.{ }^{-1}\right)$, (b) 3PA $\left(\eta \sigma_{3}\right.$, deep blue) and 4PA ( $\eta \sigma_{4}$, light blue) action cross section values of MOFs 1-6. The gray bars indicate the experimental error.

leading to a positively charged TCPE. Here, the choice of $\mathrm{Zr}$ versus $\mathrm{Hf}$ shows no effect on the charge transfer. For the kagome lattice Zr-MOF 4 with hydroxide co-ligands, the charge transfer is equal to $\mathbf{1}$ and 2 ( $1 e^{-}$per TCPE). However, if an electronwithdrawing TFA group is connected to the metal node of $\mathrm{Zr}-\mathrm{MOF}$ 3 , the charge transfer increases to $1.2 e^{-}$, while the conformation and strain of the incorporated TCPE is hardly affected. Thus, the only significant difference between $\mathbf{3}$ and $\mathbf{4}$ is the enhanced charge transfer in $\mathbf{3}$ caused by the TFA co-ligand. In line with design criterion a) above, we thus attribute the increased MPA of the top performing MOF 3 (Figure 2) to this enhanced polarization of the chromophore.

In addition to the charge transfer, the TCPE linker in a MOF is strained and constrained in a plane. According to design criterion a) an increased charge polarization of a chromophore molecule enhances its NLO properties. $\left.{ }^{[5 b}, 12,19\right]$ However, it is not immediately clear how the charge polarization of a molecule is affected by strain. To assess this, we again use computed Hirshfeld charges and focus on the neutral and the positively charged free $\mathrm{H}_{4}$ TCPE molecule as simple model for the MOFincorporated TCPE linker. Specifically, we consider the difference of the Hirshfeld charges on the inner tetraphenylethylene group and the Hirshfeld charges on the four outer phenyl carboxylic acid groups as an effective measure of a desirable multipolar charge distribution. With a net negative charge on the inner group and a net positive charge on the outer groups, already the free $\mathrm{H}_{4} \mathrm{TCPE}$ molecule exhibits such a multipolarity. To mimic the conformational strain of the TCPE linker caused by binding to the metal nodes within a MOF, we now vary the $\mathrm{C}-\mathrm{C}$ distances between the carboxylate carbon atoms in the range between 12 $\AA$ and $19 \AA$ as illustrated in Figure 4. In MOFs 1-6 the carboxylate groups of the TCPE linker are fixed in one common plane by the metal nodes. To account for this, we keep the $\mathrm{CO}_{2} \mathrm{H}$ groups fixed in the $x y$-plane, while the remaining $\mathrm{H}_{4}$ TCPE molecule is fully relaxed. Figure $4 \mathrm{a}$ shows that the charge polarization as measured by the Hirshfeld charge difference is indeed strongly affected by the conformational strain. For both the neutral and the charge case, it exhibits a clear maximum for a C-C distance of $16 \AA$. The absolute value is furthermore a factor of three larger for the charged molecule, which we expect to better represent the TCPE linker in the MOFs. If the 2PA cross section value is indeed influenced by an increased charge polarization (design criterion a) listed above, then this would suggest highest 2PA cross section values for a C-C distance of $16 \AA$.

To our knowledge, this is the first time that the influence of strain-induced charge polarization on 2PA cross section values of chromophore molecules has been investigated. As the explicit calculation of the 2PA cross section values for a charged chromophore is an unresolved challenge, we focus here on the neutral $\mathrm{H}_{4}$ TCPE. The two-photon transition tensor elements for a transition from the ground state $\langle 0|$ to the excited state $|f\rangle$ are calculated by the sum-over-state formula

$$
S_{\alpha \beta}=\sum_{i}\left[\frac{\left\langle 0\left|\mu^{\alpha}\right| i\right\rangle\left\langle i\left|\mu^{\beta}\right| f\right\rangle}{\omega_{i}-\frac{\omega_{f}}{2}}+\frac{\left\langle 0\left|\mu^{\beta}\right| i\right\rangle\left\langle i\left|\mu^{\alpha}\right| f\right\rangle}{\omega_{i}-\frac{\omega_{f}}{2}}\right],
$$

where $\omega_{\mathrm{i}}$ is the excitation energy for the intermediate state $|\boldsymbol{i}\rangle$ and $\boldsymbol{\mu}^{\boldsymbol{\alpha}, \boldsymbol{\beta}}$ is the electronic dipole operator. For randomly oriented gasphase molecules, the 2PA cross section is given by orientational averaging over the two-photon absorption probability. ${ }^{[20]}$ Figure $4 b$ shows the three highest 2PA values and their transition energies. Comparing Figure $4 \mathrm{a}$ and Figure $4 \mathrm{~b}$, we see that the change in charge polarization as derived from the Hirshfeld charge measure indeed impacts the 2PA as expected. Exactly as found for the Hirshfeld charges, the 2PA is highest for a C-C distance of $16 \AA$ (Supporting Information, Table S10), i.e. a value that is close to the strain parameters of MOFs 1, $\mathbf{2}$ and $\mathbf{3}$ (vertical lines in Figure $4 b)$.

The 2PA values from Figure $4 \mathrm{~b}$ are calculated assuming a random orientation of $\mathrm{H}_{4}$ TCPE in the gas phase. However, the samples in this work are powders of micro-crystalline MOFs. Only these crystals are randomly oriented during the measurement, while within a MOF crystal the linkers are specifically ordered. At present, the calculation of NLO properties for periodic systems 
with up to 648 atoms is computationally prohibitive. Nevertheless, if the linkers behave near independently, then their contributions will be additive. ${ }^{[12]}$ To approximately account for the shortrange molecular order within a MOF crystal, we thus use the molecular $\boldsymbol{S}_{\alpha \beta}$ (Supporting Information, Table S10) to calculate the two-photon transition tensor elements of the unit cell in a simplified model. This model neglects any molecular correlation effects beyond their tensor elements $\boldsymbol{S}_{\alpha \beta}$, as well as the influence of the nodes and co-ligands. After summing up the corresponding tensor elements $\boldsymbol{S}_{\alpha \beta}$ of each molecule in the MOF structure, orientational averaging is applied to account for the random orientation of the MOF micro-crystals (see Supporting Information, Section 4.4).

For 1 and 2 (cubic lattice), we use $S_{\alpha \beta}$ of $\mathrm{H}_{4}$ TCPE with a C-C distance of $15 \AA$, and for 3-6 (kagome lattice) the $17 \AA$ tensor elements are used. This leads to 2PA cross section values of 35 GM/chromophore for 1 and 2 and $163 \mathrm{GM} / \mathrm{chromophore}$ for 3-6. This relative trend is in excellent agreement with our experimental findings, which differentiates the MOFs 1-6 into two groups (Figure 3). These results demonstrate the importance of the details of the linker conformation, strain, crystal structure and the arrangement of the linkers when designing MOFs for MPA applications. The MPA properties are most likely further enhanced by additional intermolecular coupling of the linkers, charge transfer through the nodes and co-ligands. Indeed, the enhanced MPA properties of some thin film materials which feature aggregated chromophores, e.g. porphyrins, were assigned to such intramolecular coupling. ${ }^{[12]}$

From our model, we deduce that the 2PA would be maximized in a MOF with a planar arrangement of the TCPE linker, as then the $S_{\alpha \beta}$ would be the sum of all molecules. A good representation of such an ideal arrangement is for example given by [ $\left.\mathrm{Zn}_{2}(\mathrm{TCPE})\right]$, a MOF known for its high quantum efficiency. ${ }^{[9 c]}$ The model thus allows to quickly evaluate existing TCPE-based MOF structures for their MPA performance. For example, we estimate 2PA cross section values of $156 \mathrm{GM} /$ chromophore for $\left[\mathrm{Cd}_{3}(\mathrm{TCPE})_{1.5}\left(\mathrm{H}_{2} \mathrm{O}\right)_{2}(\mathrm{DMF})\right]{ }^{[21]}, 165 \mathrm{GM} /$ chromophore for $\mathrm{K}\left[\mathrm{Bi}(\mathrm{TCPE})(\mathrm{DMF})_{2}\right](\mathrm{DMF}=\mathrm{N}, \mathrm{N} \text {-dimethylformamide })^{[22]}$, and 170 $\mathrm{GM} /$ chromophore for the above mentioned [ $\mathrm{Zn}_{2}(\mathrm{TCPE}){ }^{[9 \mathrm{c}]}$. Indeed, in full accordance with the model we measure a value of and MOF 3 (vertical solid line).
(1) (2)

(3)
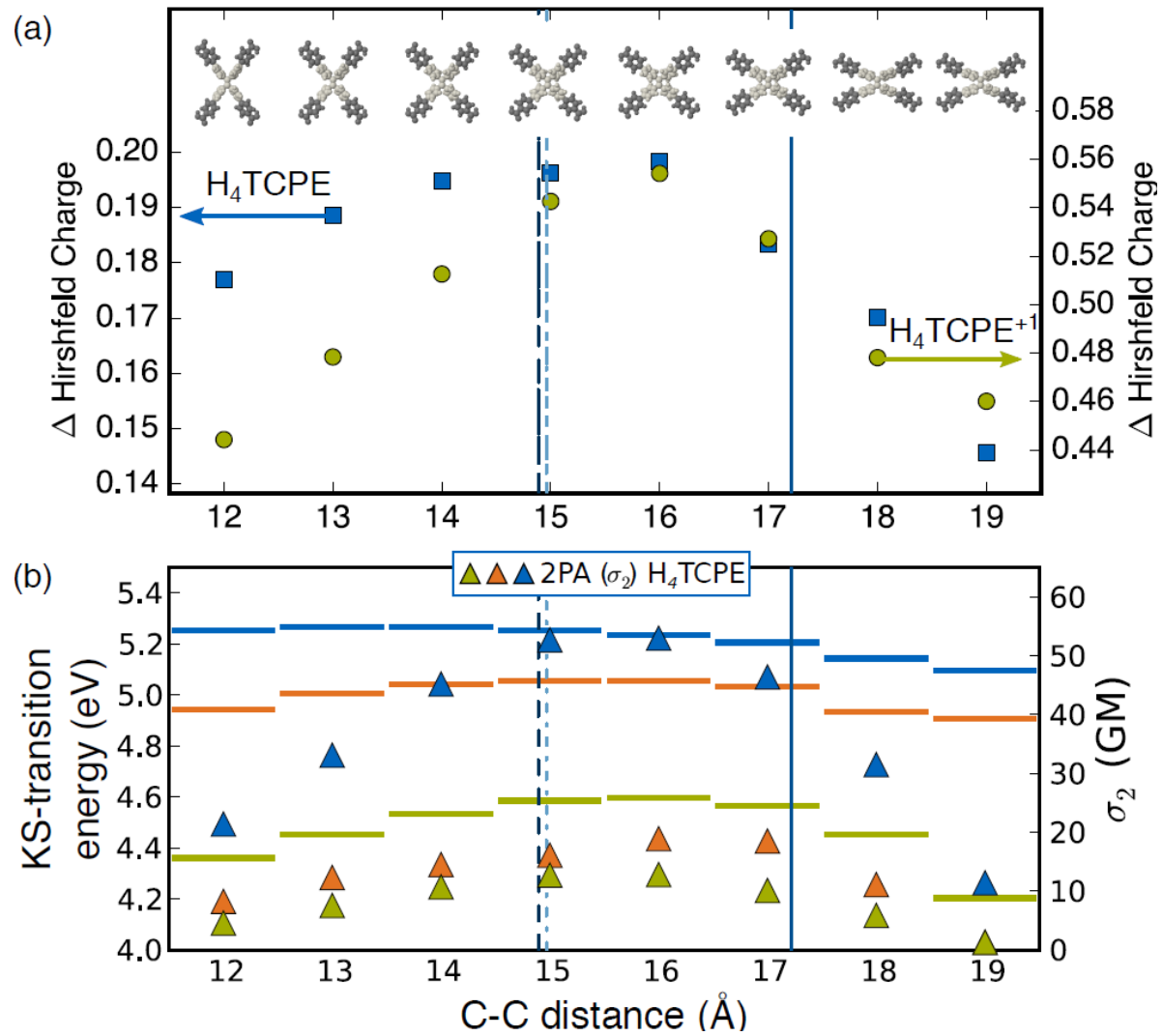

Figure 4. Calculated change in charge polarization, the symmetry allowed energy transitions and the 2PA for a free $\mathrm{H}_{4}$ TCPE molecule. As illustrated by the molecular geometries in the top row, the C-C molecular moieties are drawn with different shadings in the molecules in the top row. A positively charged $\mathrm{H}_{4} \mathrm{TCPE}^{+1}$ exhibits a three times enhanced polarization as compared to the neutral $\mathrm{H}_{4} \mathrm{TCPE}$ molecule (see text). bars; left hand axis) for the neutral molecule. Both, the Hirshfeld charge difference and the 2PA, are highest for a C-C distance of $16 \AA$, i.e. a value that falls between the strain parameters of MOFs 1, 2 (vertical dashed lines)

$4300 \mathrm{GM}$ for this latter compound, i.e. a value that even exceeds the one of our previously top-performing MOF 3 (Supporting Information, Figure S17 and Table S4). The model presented in this work is correspondingly a first step to evaluate the chromophore linker arrangement quantitatively and it can give a first indication whether a MOF is a promising candidate for MPA applications. However, if correlation effects between the chromophore linkers beyond the $S_{\alpha \beta}$ tensor elements play a dominant role, the model will not be sufficient to describe the system.

In conclusion, the modular building block principle and reticular chemistry of crystalline coordination networks, of which MOFs represent a prominent sub-class, provide unique opportunities to design crystalline structures with distinct NLO and other photo-physical properties. ${ }^{[7 b},{ }^{23]}$ Framework-integrated chromophores in MOFs show increased stability against thermal, chemical and light induced degradation, along with reduced sensitivity towards water and various solvents. ${ }^{[96,13,15]}$ The metal ions of the nodes can be chosen to facilitate the synthesis process 
and to enhance the stability of the crystal. In this contribution, we demonstrated how such MOFs can be specifically designed for MPA applications by fulfilling a range of design concepts. This allowed us to reach unprecedentedly high 2PA values. We developed a quantitative model that is useful to evaluate existing structures of MOFs and crystalline coordination networks in general. It predicts that the intrinsic MPA properties can be further increased in MOFs by maximizing the two-photon transition tensor of the unit cell. Our results show how this can be achieved by the control of the arrangement of rigidified and at the same time suitably strained NLO chromophore linkers in a high packing density together with the adjustment of charge transfer by additional co-ligands attached to the nodes.

\section{Acknowledgements}

R.M. is grateful to the Alexander von Humboldt foundation for a post-doctoral fellowship. L.N. and K.R. acknowledge funding by the German Federal Ministry of Education and Research (BMBF) within project "ELPA-AEO" (project number 01IH15001). V.N. would like to thank the Singapore Ministry of Education Academic Research Fund Tier 3 (Grant MOE2011-T3-1-005) for financial support. M.S. acknowledges NCN grant UMO2013/10/A/ST4/00114. R.A.F. acknowledges the German Research Foundation for the installation of Priority Program 1928 "COORNETs" (http://www.coornets.tum.de). The authors also would like to thank Prof. Dr. Egbert Zojer and Dr. Ashok Keerthi for fruitful discussions. R.M., L.N., V.N., K.R. and R.A.F. conceived the idea. R.M. synthesized and characterized the compounds. L.N. performed DFT and TDDFT calculations of optical properties calculations and the theoretical modeling. V.N. performed the experimental nonlinear optical studies. S.H. performed the crystallographic analyses. M.S. helped discussing the measurement procedures. L.N., R.M., K.R., R.A.F. wrote the manuscript with contributions from all the authors.

\section{Conflict of interest}

The authors declare no conflict of interest.

Keywords: metal-organic frameworks • nonlinear optics • photon upconversion $\cdot$ TDDFT $\cdot$ charge polarization

[1] F. Helmchen, W. Denk, Nat. Methods 2005, 2, 932-940.

[2] M. Wuttig, N. Yamada, Nature Mater. 2007, 6, 824-832.

[3] P.-A. Bouit, G. Wetzel, G. Berginc, B. Loiseaux, L. Toupet, P. Feneyrou, Y. Bretonnière, K. Kamada, O. Maury, C. Andraud, Chem. Mater. 2007 19, 5325-5335.

[4] S. Kawata, H. B. Sun, T. Tanaka, K. Takada, Nature 2001, 412, 697-698.

[5] a) G. S. He, L.-S. Tan, Q. Zheng, P. N. Prasad, Chem. Rev. 2008, 108, 1245-1330; b) M. Pawlicki, H. A. Collins, R. G. Denning, H. L. Anderson, Angew. Chem. Int. Ed. 2009, 48, 3244-3266; c) M. Albota, D. Beljonne, J.-L. Brédas, J. E. Ehrlich, J.-Y. Fu, A. A. Heikal, S. E. Hess, T. Kogej, M. D. Levin, S. R. Marder, D. McCord-Maughon, J. W. Perry, H. Röckel, M. Rumi, G. Subramaniam, W. W. Webb, X.-L. Wu, C. Xu, Science 1998, 281, 1653-1656.
[6] a) A. Burns, H. Ow, U. Wiesner, Chem. Soc. Rev. 2006, 35, 1028-1042 b) S. W. Thomas, G. D. Joly, T. M. Swager, Chem. Rev. 2007, 107, 13391386

[7] a) O. M. Yaghi, M. O'Keeffe, N. W. Ockwig, H. K. Chae, M. Eddaoudi, J. Kim, Nature 2003, 423, 705-714; b) H. Furukawa, K. E. Cordova, M. O'Keeffe, O. M. Yaghi, Science 2013, 341, 1230444

[8] a) H. K. Chae, D. Y. Siberio-Perez, J. Kim, Y. Go, M. Eddaoudi, A. J. Matzger, M. O'Keeffe, O. M. Yaghi, Nature 2004, 427, 523-527; b) H.-C Zhou, J. R. Long, O. M. Yaghi, Chem. Rev. 2012, 112, 673-674.

[9] a) R. Medishetty, J. K. Zareba, D. Mayer, M. Samoc, R. A. Fischer, Chem. Soc. Rev. 2017, 46, 4976-5004; b) Z. Wei, Z.-Y. Gu, R. K. Arvapally, Y.P. Chen, R. N. McDougald, J. F. Ivy, A. A. Yakovenko, D. Feng, M. A Omary, H.-C. Zhou, J. Am. Chem. Soc. 2014, 136, 8269-8276; c) Z. Hu G. Huang, W. P. Lustig, F. Wang, H. Wang, S. J. Teat, D. Banerjee, D. Zhang, J. Li, Chem. Commun. 2015, 51, 3045-3048.

[10] a) J. Yu, Y. Cui, C. Wu, Y. Yang, Z. Wang, M. O'Keeffe, B. Chen, G. Qian Angew. Chem. Int. Ed. 2012, 51, 10542-10545; b) J. Yu, Y. Cui, H. Xu Y. Yang, Z. Wang, B. Chen, G. Qian, Nature Commun. 2013, 4, 2719; c) H. He, E. Ma, Y. Cui, J. Yu, Y. Yang, T. Song, C.-D. Wu, X. Chen, B. Chen, G. Qian, Nature Commun. 2016, 7, 11087.

[11] G. S. He, Nonlinear Optics and Photonics, Oxford University Press, 2014

[12] E. Collini, Phys. Chem. Chem. Phys. 2012, 14, 3725-3736.

[13] Q. Zhang, J. Su, D. Feng, Z. Wei, X. Zou, H.-C. Zhou, J. Am. Chem. Soc. 2015, 137, 10064-10067.

[14] a) M. Rumi, J. W. Perry, Adv. Opt. Photon. 2010, 2, 451-518; b) N. S. Makarov, M. Drobizhev, A. Rebane, Opt. Express 2008, 16, 4029-4047.

[15] R. Medishetty, V. Nalla, L. Nemec, S. Henke, D. Mayer, H. Sun, K. Reuter, R. A. Fischer, Adv. Mater. 2017, 29, 1605637.

[16] K. Aidas, C. Angeli, K. L. Bak, V. Bakken, R. Bast, L. Boman, O. Christiansen, R. Cimiraglia, S. Coriani, P. Dahle, E. K. Dalskov, U. Ekström, T. Enevoldsen, J. J. Eriksen, P. Ettenhuber, B. Fernández, L. Ferrighi, H. Fliegl, L. Frediani, K. Hald, A. Halkier, C. Hättig, H. Heiberg, T. Helgaker, A. C. Hennum, H. Hettema, E. Hjertenæs, S. Høst, I.-M Høyvik, M. F. lozzi, B. Jansík, H. J. A. Jensen, D. Jonsson, P. Jørgensen, J. Kauczor, S. Kirpekar, T. Kjærgaard, W. Klopper, S. Knecht, R Kobayashi, H. Koch, J. Kongsted, A. Krapp, K. Kristensen, A. Ligabue, O. B. Lutnæs, J. I. Melo, K. V. Mikkelsen, R. H. Myhre, C. Neiss, C. B. Nielsen, P. Norman, J. Olsen, J. M. H. Olsen, A. Osted, M. J. Packer, F. Pawlowski, T. B. Pedersen, P. F. Provasi, S. Reine, Z. Rinkevicius, T. A Ruden, K. Ruud, V. V. Rybkin, P. Sałek, C. C. M. Samson, A. S. de Merás, T. Saue, S. P. A. Sauer, B. Schimmelpfennig, K. Sneskov, A. H. Steindal, K. O. Sylvester-Hvid, P. R. Taylor, A. M. Teale, E. I. Tellgren, D. P. Tew, A. J. Thorvaldsen, L. Thøgersen, O. Vahtras, M. A. Watson, D. J. D. Wilson, M. Ziolkowski, H. Ågren, WIREs Comput. Mol. Sci. 2014, 4, 269284.

[17] T. Yanai, D. P. Tew, N. C. Handy, Chem. Phys. Lett. 2004, 393, 51-57.

[18] F. L. Hirshfeld, Theor. Chim. Acta 1977, 44, 129-138.

[19] E. Zojer, D. Beljonne, P. Pacher, J.-L. Brédas, Chem. Eur. J. 2004, 10, 2668-2680.

[20] P. R. Monson, W. M. McClain, J. Chem. Phys. 1970, 53, 29-37.

[21] W. Yang, G. Chang, H. Wang, T.-L. Hu, Z. Yao, K. Alfooty, S. Xiang, B. Chen, Eur. J. Inorg. Chem. 2016, 2016, 4470-4475.

[22] B. J. Deibert, E. Velasco, W. Liu, S. J. Teat, W. P. Lustig, J. Li, Cryst. Growth Des. 2016, 16, 4178-4182.

[23] a) C. E. Wilmer, M. Leaf, C. Y. Lee, O. K. Farha, B. G. Hauser, J. T. Hupp R. Q. Snurr, Nature Chem. 2012, 4, 83-89; b) H. S. Quah, W. Chen, M. K. Schreyer, H. Yang, M. W. Wong, W. Ji, J. J. Vittal, Nature Commun. 2015, 6, 7954. 
Table of Contents

COMMUNICATION

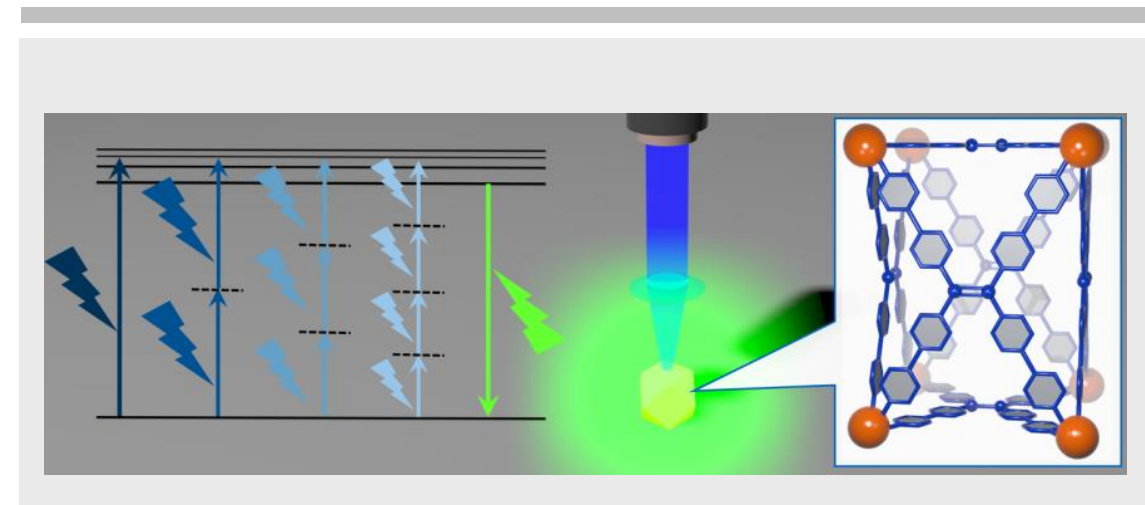

Raghavender Medishetty, ${ }^{+}$Lydia

Nemec, Venkatram Nalla, ${ }^{*}$ Sebastian Henke, Marek Samoć, Karsten Reuter, * Roland A. Fischer*

Page No. - Page No.

Multi-Photon Absorption in MetalOrganic Frameworks

Nonlinear optical properties in MOFs have been enhanced to record high multi-photon absorption cross section values by following five design criteria, supported by theory. 